\title{
A CONSISTENT DESCRIPTION OF THE SPATIAL DISTRIBUTION OF WIND GENERATED WAVES WITHIN HURRICANES
}

\author{
lan Young, University of Melbourne, ian.young@unimelb.edu.au
}

\section{INTRODUCTION}

In tropical and sub-tropical regions, hurricanes (or tropical cyclones or typhoons) represent the most extreme meteorological systems. The complex vortex structure of the winds in such storms also represent a demanding test of the physics of numerical models. Despite the apparent complexity of these systems, the fact that the intense low pressure systems are well formed means that the wind field can be parameterized with surprising accuracy. This feature of the wind field, together with extensive insitu buoy and remote sensing measurements means that a detailed understanding of the spatial distribution of the waves and the form of the directional spectrum is emerging. This paper will summarize the various data sets available and present a consistent description of the hurricane wave field suitable for coastal and ocean engineering applications.

\section{HURRICANE WAVE MEASUREMENTS}

Over the past 20 years, a number of comprehensive hurricane data sets have been obtained. These include:

(a) The detailed measurements of directional spectra from insitu buoys during the passage approximately 20 storms on the North-West coast of Australia (Young, 2006).

(b) The extensive satellite altimeter transects of wind speed and wave height through hundreds of hurricanes in all the global basins presented by Young and Vinoth (2013).

(c) The Scanning Radar Altimeter (SRA) directional spectra obtained by plane flights through US hurricanes by Hwang and Walsh (2016).

These very extensive composite datasets will be analysed to determine the spatial structure of significant wave height, as well the shape of the directional wave spectrum.

\section{SPATIAL DISTRIBUTION OF WAVES}

Two general approaches will be presented to describe the spatial distribution of significant wave height. The first of these is the "extended fetch" model of Young and Vinoth (2013). In this model, it is assumed that waves generated on the intense wind side of the hurricane move forward in the same direction as the storm. As such, they experience an "extended fetch" and the "effective fetch" for the waves becomes a function of the velocity of forward movement of the hurricane and the maximum wind velocity in the storm. Adopting this approach, it can be shown that it is possible to predict the significant wave height distribution using standard JONSWAP-type relationships.

The second approach described is the "circular racetrack" analogy of Hwang and Walsh (2016). In this description, it is proposed that the "effective fetch" increases with the distance from the center of the storm. Again, JONSWAPtype relationships can be used to determine the significant wave height. The results will, however, show that this approach is not generally applicable across a wide range of hurricane wind field parameters.

\section{SPECTRAL SHAPE}

As shown in Figure (1), the data of both Young (2006) and Hwang and Walsh (2016) show that, although hurricane wave spectra are often directionally skewed, the onedimensional form is remarkable similar to the fetch-limited JONSWAP shape. It is assumed that this is the case since nonlinear interaction terms dominate the spectral shape in these strongly forced systems.

Figure (1) shows data from both Young (2006) and Hwang and Walsh (2016). The solid line in the figure is the fetchlimed JONSWAP relation. The agreement is remarkable.

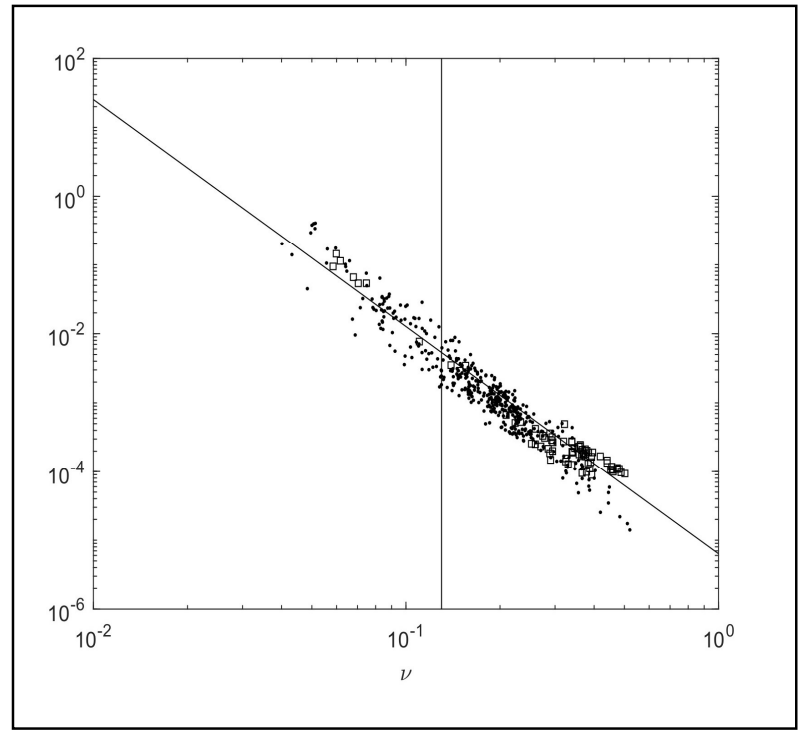

Figure 1: Non-dimensional fetch verses non-dimensional frequency for hurricane data. The solid line is the fetchlimited JONSWAP relationship.

The paper will show that all of the spectral parameters for hurricane wave spectra are consistent with JONSWAP relationships. As a result, it is possible to not only parameterize the spatial distribution of hurricane waves, but also the detailed spectral shape.

The analysis will also describe the directional distribution of the waves, where the combined data sets again present a consistent picture of the directional structure.

\section{REFERENCES}

Hwang and Walsh (2016): Azimuthal and Radial Variation of Wind-Generated Surface Waves inside Tropical Cyclones, Jnl. Phys. Oceanogr., 46, 26052621.

Young (2006): Directional spectra of hurricane windwaves, J. Geophys. Res., 111, C08020

Young, and Vinoth (2013), An 'extended fetch' model for the spatial distribution of tropical cyclone windwaves as observed by altimeter, Ocean Eng., 70, 1424. 